\title{
The $\mathrm{W}, \mathrm{X}, \mathrm{Y}$ and $\mathrm{Z}$ of sex-chromosome dosage compensation
}

\author{
Judith E. Mank \\ University of Oxford, Department of Zoology, Edward Grey Institute, South Parks Road, Oxford \\ OX1 3PS, UK
}

\begin{abstract}
In species with highly differentiated sex chromosomes, imbalances in gene dosage between the sexes can affect overall organismal fitness. Regulatory mechanisms were discovered in several unrelated animals, which counter gene-dose differences between females and males, and these early findings suggested that dosage-compensating mechanisms were required for sexchromosome evolution. However, recent reports in birds and moths contradict this view because these animals locally compensate only a few genes on the sex chromosomes, leaving the majority with different expression levels in males and females. These findings warrant a re-examination of the evolutionary forces underlying dosage compensation.
\end{abstract}

\section{Sex-chromosome differentiation creates an unbalanced gene dose}

The process of sex-chromosome divergence has profound effects on gene content. In male heterogametic lineages (those in which females are XX and males XY), mutational silencing and deletions gnaw away at an increasing proportion of the genes on the $\mathrm{Y}$ chromosome [1]. As a result, the sexes experience an imbalanced gene dose (see Glossary) because females have two $\mathrm{X}$ chromosomes and, therefore, two copies of $\mathrm{X}$-linked genes, whereas males have only a single copy on their sole $\mathrm{X}$ chromosome. This difference in X-chromosome dose, if not countered, leads to higher transcription levels for X-linked genes [2,3] in females than in males (Box 1). In species with female heterogametic sex chromosomes (those in which females are ZW and males ZZ), a similar degradation of the $\mathrm{W}$ chromosome leads to higher transcription levels in males than females for Z-linked genes. Pronounced differences between the sex chromosomes can affect the dosage of hundreds, even thousands of genes, and these effects would be transmitted upward via gene pathways and networks throughout the entire transcriptome [4].

Different transcription levels for sex-linked genes, and for the genes they control, ultimately translates to altered protein levels. Many biological processes require precise protein titres [5] and although females and males clearly differ at the transcriptional level in many ways [6-8], the majority of biochemistry does not vary by sex. Many X- and Z-linked genes contribute to essential cellular processes, and the difference in gene dose could therefore produce serious phenotypic consequences for numerous traits, potentially imposing a severe cost to the heterogametic sex.

The presence of sex chromosomes in so many animals suggests that mechanisms must be in place to mitigate the effects of gene-dose differences for sex-linked genes, and the discovery of complex sex-chromosome dosage-compensating mechanisms in several unrelated animal lineages confirms this hypothesis. However, recent studies have complicated our understanding of sex-chromosome dosage compensation, suggesting that there is far more

(C) 2009 Elsevier Ltd. All rights reserved.

Corresponding author: Mank, J.E. (judith.mank@zoo.ox.ac.uk). 
diversity in this regulatory process than previously assumed. These new data show that some groups of animals possess sex chromosomes without accompanying dosage compensating mechanisms. This indicates that complex regulatory mechanisms countering the effects of gene dose for the entire sex chromosome might not in fact be required for life, and begs the question as to why such machinery exists at all.

\section{The old paradigm of sex-chromosome dosage compensation}

As with many biological phenomena, initial studies of sexchromosome dosage compensation were confined to model organisms: eutherian mammals (mice and humans), Drosophila melanogaster, and Caenorhabditis elegans. These studies observed a mechanism in each group of animals that regulates the transcriptional activity of the entire sex chromosome, with the result being equalized male and female expression levels (Box 2). Interestingly, although the results were virtually the same across these species, the actual mechanisms of dosage compensation were very different. The differences among these global dosage-compensating mechanisms underscore the fact that the sex chromosomes of eutherian mammals, flies and nematodes represent three independent origins of male heterogamety, and so the dosage-compensating mechanisms that accompany them are evolutionarily convergent as well.

\section{Box 1. Sex-chromosome divergence creates an unbalanced gene dose between males and females}

Sex chromosomes diverge owing to the suppression of recombination between them in the heterogametic sex. In male heterogametic lineages, this suppression of recombination causes the gene content of the $\mathrm{Y}$ chromosome to degrade because genes that are not crucial for male fitness are subject to accelerated rates of gene silencing and deletion $[1,56]$ (Figure Ia). This process creates an imbalance in gene dose between females and males for X-linked genes because females have two copies of the $\mathrm{X}$ and males have only one. As recombination suppression spreads along the length of the $\mathrm{Y}$ chromosome over evolutionary time, a greater proportion of the $\mathrm{X}$ chromosome is affected by gene-dose differences between the sexes. An analogous process of $\mathrm{W}$ chromosome degradation in female heterogametic lineages results in two copies of Z-linked genes in males and only one in females.

In the absence of global dosage-compensating mechanisms, the difference in Xchromosome dose results in pronounced female-bias for the affected genes (Figure Ib). The extra gene copy presents a second target for transcriptional machinery in females, resulting in higher expression levels for these genes in females than in males. For female heterogametic systems, the difference in Z-chromosome dosage produces greater expression in males than females. Interestingly, autosomal gene duplication studies indicate that a doubled gene dose does not usually result in double transcription, but rather a 1.4-1.5-fold increase [2,3].This might be the result of the regulatory or buffering effects of gene networks [14]. 


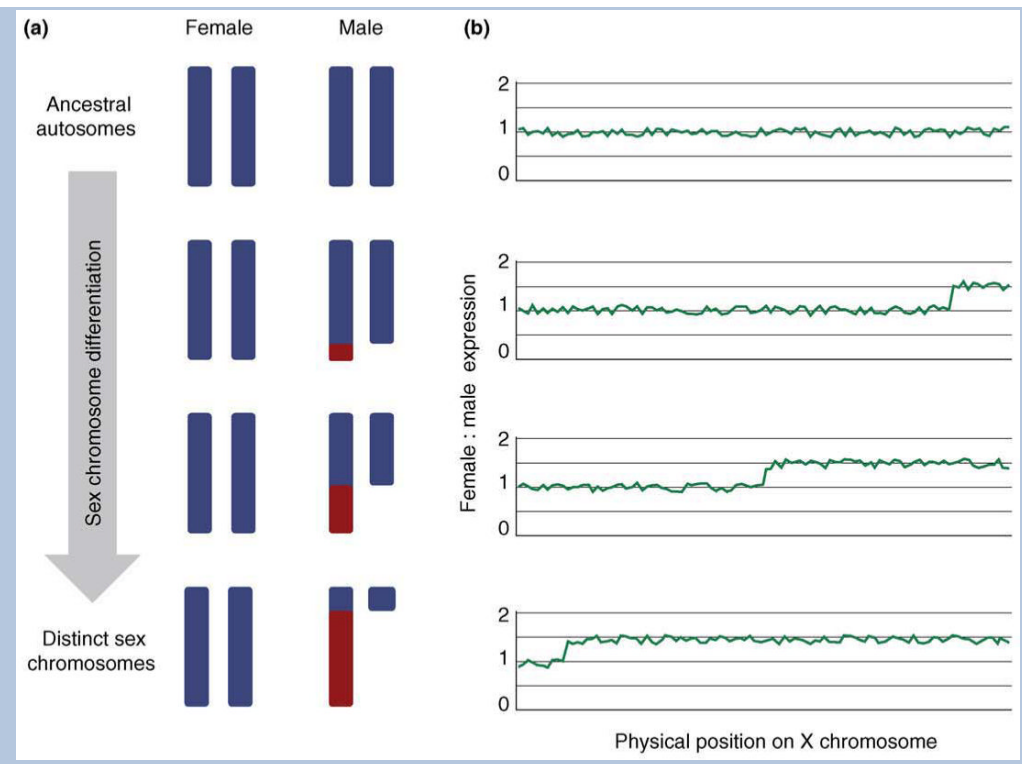

Figure I.

Chromosome divergence causes gene-dose differences between the sexes. (a) As the Ychromosome-coding content degrades, an increasing number of genes on the $\mathrm{X}$ chromosome are present in different doses in males and females (shown in red). (b) This difference in gene dose ultimately causes gene transcription differences between the sexes.

Regulating the expression of one or a small cluster of genes is accomplished thousands of times per second in complex organisms and, although remarkable, it occurs through a comparatively straightforward process. The transcriptional regulation of an entire chromosome is far more complex, requiring regulatory factors to work in concert across a large distance, and the discovery of three convergent and astonishingly complicated regulatory machineries led to the reasonable conclusion that global dosage compensation was a necessary regulatory companion of Y-chromosome degeneration. Further circumstantial evidence to support this conclusion came from observations of the deleterious effects of dosage variation for many autosomal genes and chromosomes [9]. This reasoning was extended to systems with female heterogamety with the assumption that global dosagecompensating mechanisms, or mechanisms that regulate all the genes across the sex chromosome together, would also accompany $\mathrm{W}$-chromosome degradation.

\section{Box 2. Dosage-compensating mechanisms in mammals, flies and nematodes}

The mechanism of X-chromosome dosage compensation in therian mammals is the most complex yet described. Early in development, one X chromosome in the female embryo is inactivated, a process that is less complete in mice than in humans. In eutherians, either the male or the female $\mathrm{X}$ chromosome can be inactivated and the resulting female is chimeric because some of her cells express the maternal $\mathrm{X}$ and the others express the paternal X [15]. In marsupials [32], and the placental tissue of mice [34,35] and cattle [36], the paternal $X$ is always inactivated. Regardless of the parent of origin, $X$ inactivation ensures that, although a handful of genes escape inactivation [57], transcription is equalized between males and females for the majority of genes on the $\mathrm{X}$. Transcription of the single active $\mathrm{X}$ chromosome is then upregulated in both sexes [37] 
(Figure Ia) such that expression of these genes matches average expression of genes on the diploid autosomes $(\mathrm{X}: \mathrm{A}$ transcription ratio $=1)$.

Invertebrates possess slightly different mechanisms, but the results are equivalent. The mechanism of global dosage compensation is simplest in Drosophila (Figure 1b), in which hyper-transcription of the single male $\mathrm{X}$ chromosome simultaneously equalizes expression between the sexes and achieves parity between the $\mathrm{X}$ chromosome and autosomes $(\mathrm{X}: \mathrm{A}=1)[43,44]$. Hyper-transcription of the $\mathrm{X}$ occurs in both hermaphrodites and males in $C$. elegans [45] (Figure Ic). This process equalizes X:A transcription in males but overshoots it in hermaphrodites $(\mathrm{X}: \mathrm{A}>1)$ because both $\mathrm{X}$ chromosomes are hyper-transcribed. A simultaneous hermaphrodite-specific global dosage-compensating mechanism counters the hyper-transcription, and returns X:A transcription to 1 [46,47].

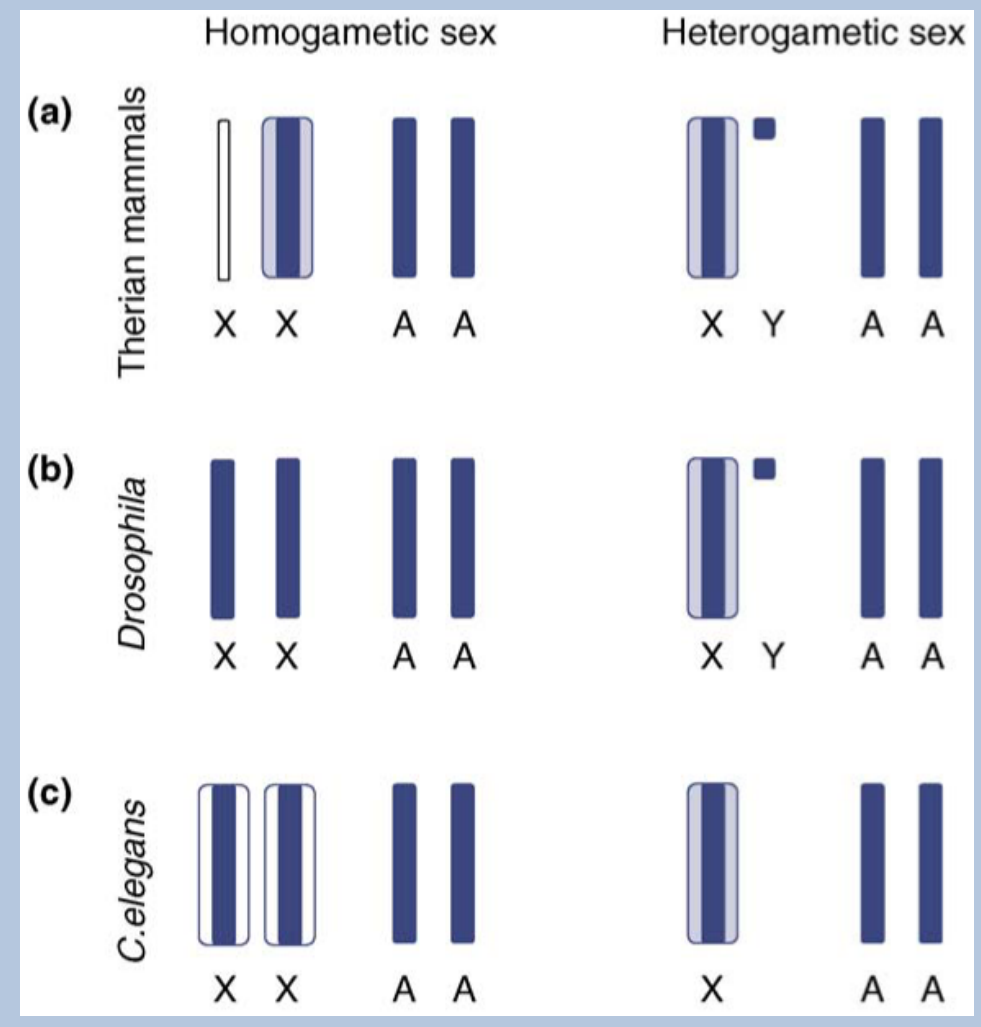

Figure I.

Global dosage-compensating mechanisms. Gene expression levels are indicated by chromosome width, levels of expected normal expression are indicated in dark blue, and hyper-transcription above this level is indicated in light blue. (a) In therian mammals, one $\mathrm{X}$ is inactivated in females (indicated by the thin white line) [15], then the active $\mathrm{X}$ is hyper-transcribed in both sexes to equalize expression levels with autosomal genes [37]. (b) In Drosophila, parity between the sexes is achieved through hyper-transcription of the male $\mathrm{X}$ chromosome, a process that also equalizes expression between the $\mathrm{X}$ and the autosomes [43,44]. (c) In C. elegans, hyper-transcription occurs for X chromosomes in both sexes [45], achieving X:A parity in males. However, X:A >1 in hermaphrodites, and a second hermaphrodite-specific chromosome-wide countering mechanism returns transcription in this sex to X:A parity (shown in white) [46,47]. 


\section{New evidence indicates that global dosage compensation is not necessary}

Two independent studies $[10,11]$ therefore caused some consternation when they recently reported that birds lack chromosome-wide dosage compensation. The majority of this work was based on chickens (Gallus gallus) because these are the primary avian model, and the pattern was confirmed in zebra finch (Taeniopygia guttata) [11]. Follow-up work found evidence of a small, confined area on the chicken $\mathrm{Z}$ chromosome that might be compensated [12]; however, it is possible that this is simply an artefact of a few clustered genes with expression confined to females [13]. Outside of this limited region, both studies [10,11] suggested that, although some genes were individually equalized, or locally dosagecompensated, hundreds of genes along the $\mathrm{Z}$ chromosome are expressed less in females, and female birds seem none the worse for it.

Biological paradigms often have a few exceptions in non-model organisms, and that seemed to be the case with the lack of global dosage compensation in birds. Discussions of the avian pattern attempted to explain the exception to the rule of sex-chromosome dosage compensation as the product of some hidden bird idiosyncrasy to maintain the generality of the theory $[14,15]$. However, new work suggests that birds are not at all unique in their local approach to dosage compensation. The silkworm (Bombyx mori), which, like birds, is female heterogametic, lacks a global dosage-compensating mechanism for the $\mathrm{Z}$ chromosome [16]. Again, like birds, the silkworm equalizes transcription between the sexes locally for some genes when and where needed, but hundreds of other Z-linked genes are male-biased (Figure 1), with no obvious deleterious consequences for females. Limited evidence in related genera [17] supports the conclusion that the orthologous sex chromosomes in other Lepidopteran species are not globally dosage-compensated.

\section{What the birds and Lepidopterans reveal}

There are now five independent sex-chromosome systems in which the chromosome-wide patterns of dosage compensation have been assessed, and two of these systems lack global equalizing mechanisms. Although it was possible to dismiss birds as strange, dismissing $40 \%$ of the data is simply not tenable. The discovery of a second clade lacking global dosage compensation forces us to face the fact that chromosome-wide regulatory equalizing mechanisms need not accompany sex-chromosome divergence.

The data from birds and silkworms tell us that, although it is important to equalize transcription for some crucial genes on the sex chromosomes, this can be done locally on a gene-by-gene basis, leaving the remainder of the genes with different transcription levels between the sexes (Figure 2). Moreover, because Lepidoptera and Aves both have female heterogametic sex chromosomes, the evidence in hand indicates that global dosage compensation might be confined to the male heterogametic sex chromosomal systems.

\section{Why has global dosage compensation evolved in some animals and not others?}

Just as the new findings from female heterogametic animals shatter a long-held paradigm, they also raise new and interesting questions. Some genes must be regulated more tightly than others, and copy number variation studies indicate that gene-dose differences are tolerable for a large fraction of genes [18-20]. Additionally, regulatory machinery exists to control gene expression locally in a sex-specific manner, as demonstrated by the prevalence of sex-biased gene expression from autosomal loci across the metazoans [6-8]. 
Although it is certainly a more tedious process to equalize expression in the heterogametic sex locally for crucial genes along the sex chromosome, the machinery to do this was in place before sex chromosomes began to diverge. Evolution tends to work in a pathdependent and tedious manner, fine-tuning past adaptations to new purposes more often than building new adaptations from scratch. Therefore, it can be reasoned that selection for dosage compensation of a few crucial genes would act on available machinery rather than produce an entirely novel mechanism to regulate a whole chromosome simultaneously. By this reasoning, birds and Lepidopterans therefore represent the most parsimonious approach to countering the effects of gene-dose differences. Some fraction of genes on therian, nematode and Drosophila X chromosomes must be dosage-sensitive and, although the regulation of these genes could have provided the starting point for full-chromosome regulation [21], it is not immediately clear why regulation of these crucial genes spread along the entire chromosome to regulate all the other genes that are not dosage-sensitive. In other words, because global dosage-compensating mechanisms are neither trivial nor required, why have these extraordinarily complicated mechanisms evolved convergently in mammals, flies and nematodes?

Clades that compensate gene dose globally and those that do it locally display few distinguishing biological characteristics. Each category contains vertebrates and invertebrates, large and small sex chromosomes, and different mechanisms of sex determination (Table 1). At this point, the only distinguishing characteristic seems to be sex chromosome type, given that birds and Lepidopterans are female heterogametic, and mammals, flies and nematodes are male heterogametic. However, continuing work on dosage compensation in the platypus (Ornithorhynchis anatinus) [22], discussed later, could ultimately remove this distinction as well. Is there some aspect of sex-chromosome type that explains the need for global dosage compensation?

\section{Is dosage compensation a function of the age of the sex chromosomes?}

The simplest possible explanation for the lack of global dosage compensation in some lineages is that they are too young to have evolved complex mechanisms to compensate for gene dosage along the entire chromosome. If this is the case, then it would be expected that the globally dosage-compensated sex chromosomes, specifically those of mammals, nematodes and flies, are older than those that compensate locally (i.e. birds and Lepidoptera).

The sex chromosomes of therian mammals date to at least 166 million years ago (MYA) [23,24]. Drosophila sex chromosomes are, despite some turnover, orthologous, dating at least to the origin of the genus approximately 65 MYA [25]. Avian sex chromosomes are shared by all extant birds, suggesting they originated at least 150 MYA [26,27], and possibly much earlier [28]. Lepidopteran sex chromosomes, although showing some turnover, date from 190 MYA [17]. Although the murky phylogeny of nematodes makes it difficult to pinpoint the age of Caenorhabditis sex chromosomes [29], it is clear that age does not dictate the observed pattern of dosage compensation.

\section{Dosage compensation might not explain all aspect of sex-chromosome regulation}

The phenomenon of random X-inactivation in therian mammals might be unrelated to dosage compensation. Theoretical work suggests that the process of X-inactivation could have resulted from the sexual antagonism as it plays out in the developing foetus [30,31].Thismodel predicts that the evolution of internal gestation leads to the inactivation of the paternal X chromosome as the mother attempts to reduce the higher transcription of 
$\mathrm{X}$-linked foetal growth genes in female offspring that favour the reproductive interests of the father over her own. This pattern progresses over evolutionary time-scales to random Xchromosome inactivation in response to selection to reduce the cost of functional hemizygosity in females.

When first proposed, this theoretical treatment seemed far-fetched, especially given that a perfectly feasible explanation for $\mathrm{X}$-inactivation, namely global dosage compensation, already existed. However, the new evidence from birds and silkworms requires a reexamination of this process, and the data provide some surprising support for the theory. Specifically, whereas the process of X-inactivation in eutherian mammals randomly affects either the maternal or paternal copy, X-inactivation in marsupials, which are the sister-clade to eutherian mammals, always affects the paternal chromosome [32,33]. Vestiges of the marsupial pattern are still discernible in some eutherian mammals. Specifically, the paternal $\mathrm{X}$ is inactivated in the placental tissues of mice [34,35] and cattle [36], thereby indicating that this is the ancestral form of $\mathrm{X}$-inactivation for eutherians, whereas the random pattern seen in the embryonic tissues of eutherians is derived [32], as predicted [30,31] (Figure 3).

Additionally, $\mathrm{X}$-chromosome inactivation is accompanied by transcriptional upregulation of the $\mathrm{X}$ in both males and females to achieve X:Autosome (X:A) transcriptional parity [37], a process that by itself would be sufficient to balance out dosage if it were confined to males.

Thus, the available data support the theory that $\mathrm{X}$-chromosome inactivation is not necessarily associated with chromosome dosage compensation. However, it is not known whether or not X-chromosome inactivation evolved before or after the origin of internal gestation because both events occurred after the therian divergence from the monotremes (egg-laying mammals including the platypus). For the maternal-foetal conflict hypothesis to be correct, internal gestation must have preceded X-inactivation.

\section{Is dosage compensation associated with male heterogamety?}

Regardless of whether or not mammalian X-inactivation is associated with dosage compensation or maternal-foetal conflict, mammals, flies and nematodes all have regulatory mechanisms that hyper-transcribe the $\mathrm{X}$ chromosome, thus equalizing the $\mathrm{X}$ :A transcription ratio in males [38]. Birds and Lepidopterans do this for some genes on the $\mathrm{Z}$, but the average female $\mathrm{Z}: \mathrm{A}$ ratio is $\approx 0.8$ [11]. The available five whole-chromosome data points therefore suggest that global dosage compensation, rather than being a necessary companion for sexchromosome evolution in general, is associated primarily with male heterogamety. Is there some aspect of male heterogamety that explains the observed pattern in the data?

\section{Is dosage compensation related to sexual antagonism?}

One possible reason for the pattern of global and local dosage compensation is based on sexual antagonism, which might play out differently in male and female heterogametic systems [36]. As a result of conflicting selection pressures in males and females, sexually antagonistic genes benefit one sex at the expense of the other. The sex-biased inheritance pattern of the $\mathrm{X}$ and $\mathrm{Z}$ chromosomes is predicted to render them a hotspot for sexually antagonistic genes [39]. Specifically, because the $\mathrm{X}$ is $2 / 3$ of the time in females, it is more often selected for its effects on the female phenotype. Therefore, it should harbour more genes with female-specific benefits than would be expected by chance alone. By analogy, the $\mathrm{Z}$ will harbour an excess of genes with male benefits. Because sexual antagonism can result in sex-biased gene expression [40], which is also the side effect of gene-dose differences on the sex chromosomes, is it possible that the observed pattern of dosage compensation is a function of this sexual antagonism playing out on different types of sex chromosomes? 
Preliminary reports $[41,42]$ of the over-abundance of male-biased genes on the chicken $\mathrm{Z}$ chromosome, which is well above what would be expected owing to chance alone, invoked sexual antagonism theory rather than a lack of complete dosage compensation. However, this conclusion might have been due, at least in part, to the fact that the paradigm of obligatory global dosage compensation was so well accepted that it was difficult to consider the possibility that birds lacked it. Subsequent whole-transcriptome profiling experiments revealed that the vast majority of genes on the avian $\mathrm{Z}$ chromosome were male-biased $[10,11]$, far and above the amount expected to be under sexually antagonistic selection, thus indicating that the expression pattern arose owing to uncompensated gene dose.

Furthermore, there is no obvious reason to assume that sexual antagonism would favour dosage compensation in male-heterogametic but not female-heterogametic systems. Rather, if sexual antagonism were the main evolutionary force shaping dosage-compensating mechanisms, it would be expected to act similarly on all systems regardless of type. Therefore, there is no clear reason for assuming that sexual antagonism underlies the prevalence of global dosage compensation in male heterogametic lineages.

\section{Are males more vulnerable to the effects of gene dose?}

The term 'sex-chromosome dosage compensation' can be somewhat misleading. Dosage compensation has not evolved in response to selection for equalized dose of X-linked genes between the sexes, but rather in response to selection to equalize the average transcription rate for X-linked and autosomal genes (X:A transcription ratio $=1$ ) in the heterogametic sex. All known global mechanisms of chromosomal dosage compensation show clear signs balancing the X:A transcription ratio in males (Box 2). Regardless of the ultimate cause of $\mathrm{X}$-chromosome inactivation in therian mammals discussed earlier, the single active $\mathrm{X}$ is hyper-transcribed in both sexes such that X:A transcription $=1$ [37]. In Drosophila, X-linked genes are hyper-transcribed in males to achieve this balanced ratio, a process that does not affect females $[43,44]$.

The $\mathrm{X}$ chromosome dosage-compensating mechanisms in $C$. elegans are, at least at first glance, somewhat confusing. However, when broken down into constitutive components, they become clearer. In male nematodes, the average transcription rate for X-linked genes is equalized with that of the autosomes $(\mathrm{X}: \mathrm{A}=1)$ by hyper-transcription of $\mathrm{X}$-linked genes, a process that affects both males and hermaphrodites [45]. Hermaphrodites then counter the resulting over-transcription (X:A >1) by deploying a secondary adaptation to bring their transcription in line with diploid gene dose $[46,47]$.

When selection pressures differ between females and males, the net sum of the forces determines the fixation of the adaptation that confers the greatest sex-specific benefit [48]. For example, if the benefit to males for a given adaptation far exceeds the harm to females, the adaptation will be selected. This suggests that selection to equalize X:A transcription ratio in $C$. elegans males should be stronger than selection in hermaphrodites because, at least initially, we would expect some gap in evolutionary time between the origin of $\mathrm{X}$ hyper-transcription and the counter-adaptation in hermaphrodites. Additionally, there seems to be no such need to equalize gene dose for all Z-linked genes in female birds and Lepidopterans, indicating that they are less vulnerable to the effects of heterogamety than males.

Preliminary data from the platypus, however, complicates this pattern. The monotreme sexchromosome system is somewhat peculiar, with five $\mathrm{X}$ chromosomes and five $\mathrm{Y}$ chromosomes that, although male heterogametic, share more in terms of gene content with the avian $\mathrm{Z}$ chromosome than with the convergent therian X [49,50]. Although a complete scan of the full coding content of the platypus $\mathrm{X}$ chromosomes is required, limited work on 
ten $\mathrm{X}$-linked genes outside the pseudoautosomal regions indicates that dosage compensation is present in somewhere between a half and three-quarters of genes [22]; however, statistical tests of the group of genes as a whole failed to find significant differences between male and female expression levels. Although the data could fit a range of dosage-compensation scenarios, this study suggests that dosage compensation in the platypus is intermediate: it is less than global, but greater than what is observed in birds and Lepidopterans. However, without a complete transcriptional analysis of the full platypus X-linked coding complement, it is difficult to know the exact state of platypus dosage compensation because previous work was consistent with some form of monotreme global sex-chromosome regulation [51].

\section{Concluding remarks}

Global dosage compensation is not a requisite regulatory companion of sex-chromosome divergence, and this discovery generates many interesting questions as to why it occurs at all. At this point, no single satisfying explanation for the observed pattern of dosage compensation has emerged; rather an incomplete patchwork of possibilities exists, indicating the need for much further testing (Box 3).

\section{Box 3. Outstanding questions}

Further studies of the evolution of global and local sex-chromosome dosage compensation can proceed on several fronts:

- Information on the status of dosage compensation is needed from many more instances of independently evolved sex chromosomes to establish general patterns. Preliminary data on the platypus [22] is just the beginning, and future efforts on more sex-chromosome systems will be required to determine how common global dosage compensation is, and whether or not it is truly associated with male heterogamety. Fish might prove an ideal system because there are many independent instances of both female- and male-heterogametic sex chromosomes [58], with rapid turnover of sex-chromosome types [59], within the clade.

- A finer-scale analysis of those genes that are dosage compensated and those that are not is required. In species with local compensation, genes that display equalized transcription have different broad functions than those that do not $[13,16]$. However, this type of analysis is based on databases of gene function such as the Gene Ontology (GO) [60], and the GO annotations are often generalized and largely incomplete for non-model organisms. Therefore, GO studies might miss important details in non-model animals, rendering it difficult to draw conclusions about specific types of gene function that are more or less likely to be dosage compensated. Therefore, further detailed molecular genetic information about gene function will be needed to understand why some genes display equalized transcription and others do not.

- Genetic interactions should be considered. Genes do not function in isolation and information about genetic pathways and interactions might also be needed to understand the pattern of local dosage compensation. Specifically, the location of a gene in a network (either at the fringe or in the centre) or a pathway (either at the top, bottom, or middle) might either exacerbate or buffer gene dose effects for sex-linked genes. Catalogues of genetic pathways [61] and interaction networks [62] are increasing in coverage, and these could prove 
useful in distinguishing those genes that are dosage compensated from those that are not.

Observations from five independent sex-chromosome systems are only slightly better than three, and so most important to understanding the evolution of dosage compensation is the need for information from other animals. Sex chromosomes of both inheritance patterns have evolved many times in the animal kingdom, and it is only by widening our perspective that we can understand why complex global sex-chromosome regulation evolves for some lineages and not for others, and to determine the ultimate evolutionary pressures shaping this adaptation.

\section{Glossary}

\section{Dosage- compensating mechanism}

Female heterogamety

Gene dose

Hypertranscription

Male heterogamety

Monotremes

Sex-biased gene expression

Therian mammals

Transcriptome

X-chromosome inactivation

$X: A$ transcription ratio

\author{
Z:A transcription \\ ratio
}

a regulatory mechanism to equalize transcription in response to differences in gene dose. Dosage-compensating mechanisms can regulate individual genes (local) or entire chromosomes (global).

a sex chromosome system in which the male has two $\mathrm{Z}$ chromosomes and the female one $\mathrm{Z}$ and one $\mathrm{W}$. Animals with this type of sex chromosome inheritance include birds and butterflies and some snakes and fish.

the number of copies present in the genome for a given proteincoding locus.

a regulatory process by which the rate of gene transcription is increased at a locus.

sex chromosome system in which the female has two $\mathrm{X}$ chromosomes, and the male one $\mathrm{X}$ and one $\mathrm{Y}$. Animals with this type of sex chromosome inheritance include mammals, Drosophila and $C$. elegans.

egg laying mammals, including platypus and echidnas.

genes transcribed at a higher level in one sex. Without compensating mechanisms, $\mathrm{X}$-chromosome-dose differences result in female-biased gene expression, and Z-chromosome-dose differences in male-biased gene expression.

eutherian (placental) mammals and marsupials.

the complete protein-coding content of a genome.

a process in female mammals whereby one copy of the $\mathrm{X}$ chromosome is transcriptionally silenced.

the average transcription of $\mathrm{X}$-linked genes divided by the average transcription of autosomal genes. In the absence of global dosage compensation, $\mathrm{X}: \mathrm{A}<1$ in males and $=1$ in females. Where dosage has been compensated, $\mathrm{X}: \mathrm{A}=1$ in both sexes.

the average transcription of Z-linked genes divided by the average transcription of autosomal genes. In the absence of global dosage compensation, $\mathrm{Z}: \mathrm{A}<1$ in females and $=1$ in males. Where dosage has been compensated, $\mathrm{Z}: \mathrm{A}=1$ in both sexes. 


\section{References}

1. Charlesworth B. Model for the evolution of Y chromosomes and dosage compensation. Proc. Natl. Acad. Sci. U. S. A. 1978; 75:5618-5622. [PubMed: 281711]

2. Hyman E, et al. Impact of DNA amplification on gene expression patterns in breast cancer. Cancer Res. 2002; 62:6240-6245. [PubMed: 12414653]

3. Pollack JR, et al. Microarray analysis reveals a major direct role of DNA copy number alteration in the transcriptional program of human breast tumors. Proc. Natl. Acad. Sci. U. S. A. 2002; 99:12963-12968. [PubMed: 12297621]

4. Jeong H, et al. Lethality and centrality in protein networks. Nature. 2001; 411:41-42. [PubMed: 11333967]

5. Batada NN, Hurst LD. Evolution of chromosome organization driven by selection for reduced gene expression noise. Nat. Genet. 2007; 39:945-949. [PubMed: 17660811]

6. Mank JE, et al. The unique genomic properties of sex-biased genes: Insights from avian microarray data. BMC Genomics. 2008; 9:148. [PubMed: 18377635]

7. Parisi M, et al. A survey of ovary-, testis-, and soma-biased gene expression in Drosophila melanogaster adults. Genome Biol. 2004; 5:R40. [PubMed: 15186491]

8. Yang X, et al. Tissue-specific expression and regulation of sexually dimorphic genes in mice. Genome Res. 2006; 16:995-1004. [PubMed: 16825664]

9. Hassold T, et al. The origin of human aneuploidy: where we have been, where we are going. Hum. Mol. Genet. 2007; 16:R203-R208. [PubMed: 17911163]

10. Ellegren $\mathrm{H}$, et al. Faced with inequality: chicken does not have general dosage compensation of sex-linked genes. BMC Biol. 2007; 5:40. [PubMed: 17883843]

11. Itoh Y, et al. Dosage compensation is less effective in birds than in mammals. J. Biol. 2007; 6:2. [PubMed: 17352797]

12. Melamed E, Arnold AP. Regional differences in dosage compensation on the chicken $Z$ chromosome. Genome Biol. 2007; 8:R202. [PubMed: 17900367]

13. Mank JE, Ellegren H. All dosage compensation is local: gene by gene regulation of sex-biased expression on the chicken Z chromosome. Heredity. 2009; 102:312-320. [PubMed: 18985062]

14. Oliver B. Sex, dose, and equality. PLoS Biol. 2007; 5:e340. [PubMed: 18162049]

15. Payer B, Lee JT. X chromosome dosage compensation: how mammals keep the balance. Annu. Rev. Genet. 2008; 42:733-772. [PubMed: 18729722]

16. Zha $X$, et al. Dosage analysis of $Z$ chromosome genes using microarray in silkworm, Bombyx mori. Insect Biochem. Mol. Biol. 2009 DOI: 10.1016/j.ibmb.2008.12.003.

17. Traut W, et al. Sex chromosomes and sex determination in Lepidoptera. Sex Dev. 2007; 1:332346. [PubMed: 18391545]

18. Beckmann JS, et al. Copy number variants and genetic traits: closer to the resolution of phenotypic to genotypic variability. Nat. Rev. Genet. 2007; 8:639-646. [PubMed: 17637735]

19. Birchler JA, et al. Dosage-dependent gene regulation in multicellular eukaryotes: Implications for dosage compensation, aneuploid syndromes, and quantitative traits. Dev. Biol. 2001; 234:275288. [PubMed: 11396999]

20. Mileyko Y, et al. Small-scale copy number variation and large-scale changes in gene expression. Proc. Natl. Acad. Sci. U. S. A. 2008; 105:16659-16664. [PubMed: 18946033]

21. Graves JAM, Disteche CM. Does gene dosage really matter? J. Biol. 2007; 6:1. [PubMed: 17381829]

22. Deakin JE, et al. The status of dosage compensation in the multiple $\mathrm{X}$ chromosomes of platypus. PLoS Genet. 2008; 4:e1000140. [PubMed: 18654631]

23. Bininda-Emonds OR, et al. The delayed rise of present-day mammals. Nature. 2007; 446:507-512. [PubMed: 17392779]

24. Potrzebowski L, et al. Chromosomal gene movements reflect the recent origin and biology of the therian sex chromosomes. PLoS Biol. 2008; 6:e80. [PubMed: 18384235]

25. Koerich LB, et al. Low conservation of gene content in the Drosophila Y chromosome. Nature. 2008; 456:949-951. [PubMed: 19011613] 
26. Mank JE, Ellegren H. Parallel divergence and degredation of the avian W sex chromosome. Trends Ecol. Evol. 2007; 22:389-391. [PubMed: 17573147]

27. Stiglec R, et al. A new look at the evolution of avian sex chromosomes. Cytogenet. Genome Res. 2007; 117:103-109. [PubMed: 17675850]

28. Kawai A, et al. The ZW sex chromosomes of Gekko hokouensis (Gekkonidae, Squamata) represent highly conserved homology with those of avian species. Chromosoma. 2009; 118:43-51. [PubMed: 18685858]

29. Cutter AD. Divergence times in Caenorhabditis and Drosophila inferred from direct estimates of the neutral mutation rate. Mol. Biol. Evol. 2008; 25:778-786. [PubMed: 18234705]

30. Engelstadter J, Haig D. Sexual antagonism and the evolution of X chromosome inactivation. Evolution. 2008; 62:2097-2104. [PubMed: 18507742]

31. Haig D. Self-imposed silence: parental antagonism and the evolution of X-chromosome inactivation. Evolution. 2006; 60:440-447. [PubMed: 16637489]

32. Cooper DW, et al. X inactivation in marsupials and monotremes. Semin. Dev. Biol. 1993; 4:117128.

33. Namekawa SH, et al. Sex chromosome silencing in the marsupial male germ line. Proc. Natl. Acad. Sci. U. S. A. 2007; 104:9730-9735. [PubMed: 17535928]

34. Okamoto I, et al. Evidence for de novo imprinted X-chromosome inactivation independent of meiotic inactivation in mice. Nature. 2005; 438:369-373. [PubMed: 16227973]

35. Takagi N, Sasaki M. Preferential inactivation of the paternally derived X chromsome in the extraembryonic membranes of the mouse. Nature. 1975; 256:640-642. [PubMed: 1152998]

36. Xue F, et al. Aberrant patterns of X chromosome inactivation in bovine clones. Nat. Genet. 2002; 31:216-220. [PubMed: 12032569]

37. Nguyen DK, Disteche CM. Dosage compensation of the active X chromosome in mammals. Nat. Genet. 2006; 38:47-53. [PubMed: 16341221]

38. Straub T, Becker PB. Dosage compensation: the beginning and end of generalization. Nat. Rev. Genet. 2007; 8:47-57. [PubMed: 17173057]

39. Rice WR. Sex chromosomes and the evolution of sexual dimorphism. Evolution. 1984; 38:735742.

40. Mank JE. Sex chromosomes and the evolution of sexual dimorphism: lessons from the genome. Am. Nat. 2009; 173:141-150. [PubMed: 20374139]

41. Kaiser VB, Ellegren H. Nonrandom distribution of genes with sex-biased expression in the chicken genome. Evolution. 2006; 60:1945-1951. [PubMed: 17089978]

42. Storchova R, Divina P. Nonrandom representation of sex-biased genes on chicken Z chromosome. J. Mol. Evol. 2006; 63:676-681. [PubMed: 17031459]

43. Fagegaltier D, Baker BS. X chromosome sites autonomously recruit the dosage compensation complex in Drosophila males. PLoS Biol. 2004; 2:e341. [PubMed: 15502872]

44. Rodriguez MA, et al. Species-specific positive selection of the male-specific lethal complex that participates in dosage compensation in Drosophila. Proc. Natl. Acad. Sci. U. S. A. 2007; 104:15412-15417. [PubMed: 17878295]

45. Gupta V, et al. Global analysis of X-chromosome dosage compensation. J. Biol. 2006; 5:3. [PubMed: 16507155]

46. Ercan S, et al. X chromosome repression by localization of the $C$. elegans dosage compensation machinery to sites of transcription initiation. Nat. Genet. 2007; 39:403-408. [PubMed: 17293863]

47. McDonel P, et al. Clustered DNA motifs mark X chromosomes for repression by a dosage compensation complex. Nature. 2006; 444:614-618. [PubMed: 17122774]

48. Arnqvist, G.; Rowe, L. Sexual Conflict. Princeton University Press; 2005.

49. El-Mogharbel N, et al. DMRT gene cluster analysis in the platypus: New insights into genomic organization and regulatory regions. Genomics. 2007; 89:10-21. [PubMed: 16962738]

50. Veyrunes F, et al. Bird-like sex chromosomes of platypus imply recent origin of mammal sex chromosomes. Genome Res. 2008; 18:965-973. [PubMed: 18463302]

51. Cooper DW, et al. X-inactivation in marsupials and monotremes. Semin. Dev. Biol. 1993; 4:117128. 
52. Marshall Graves JA. Weird animal genomes and the evolution of vertebrate sex and sex chromosomes. Annu. Rev. Genet. 2008; 42:565-586. [PubMed: 18983263]

53. Marin I, Baker BS. The evolutionary dynamics of sex determination. Science. 1998; 281:19901994. [PubMed: 9748152]

54. Stothard P, Pilgrim D. Sex-determination gene and pathway evolution in nematodes. Bioessays. 2003; 25:221-231. [PubMed: 12596226]

55. Fujii T, Shimada T. Sex determination in the silkworm, Bombyx mori: A female determinant on the W chromosome and the sex-determining gene cascade. Semin. Cell Dev. Biol. 2007; 18:379388. [PubMed: 17446095]

56. Charlesworth B. The evolution of sex chromosomes. Science. 1991; 251:1030-1033. [PubMed: 1998119]

57. Johnston CM, et al. Large-scale population study of human cell lines indicates that dosage compensation is virtually complete. PLoS Genet. 2008; 4:e9. [PubMed: 18208332]

58. Mank JE, et al. Evolution of alternative sex determining mechanisms in teleost fishes. Biol. J. Linn. Soc. 2006; 87:83-93.

59. Ross JA, et al. Turnover of sex chromosomes in the stickleback fishes (Gasterosteidae). PLoS Genet. 2009; 5:e1000391. [PubMed: 19229325]

60. Gene Ontology Consortium. Gene ontology: a tool for the unification of biology. Nat. Genet. 2000; 25:25-29. [PubMed: 10802651]

61. Kanehisa M, Goto S. KEGG: Kyoto Encyclopedia of Genes and Genomes. Nucleic Acids Res. 2000; 28:27-30. [PubMed: 10592173]

62. Alexeyenko A, Sonnhammer EL. Global networks of functional coupling in eukaryotes from comprehensive data integration. Genome Res. 2009 DOI: 10.1101/gr.087528.108. 


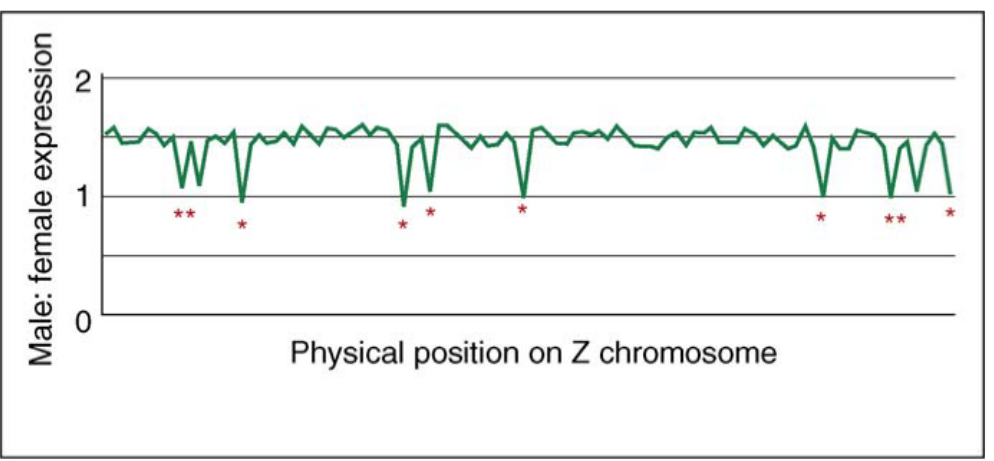

Figure 1.

Transcriptional profile for hypothetical local dosage compensation in a female heterogametic animal. Although some randomly distributed crucial genes are compensated locally $(*)$, the vast majority show 1.5 times higher expression in males as a result of genedose differences. 


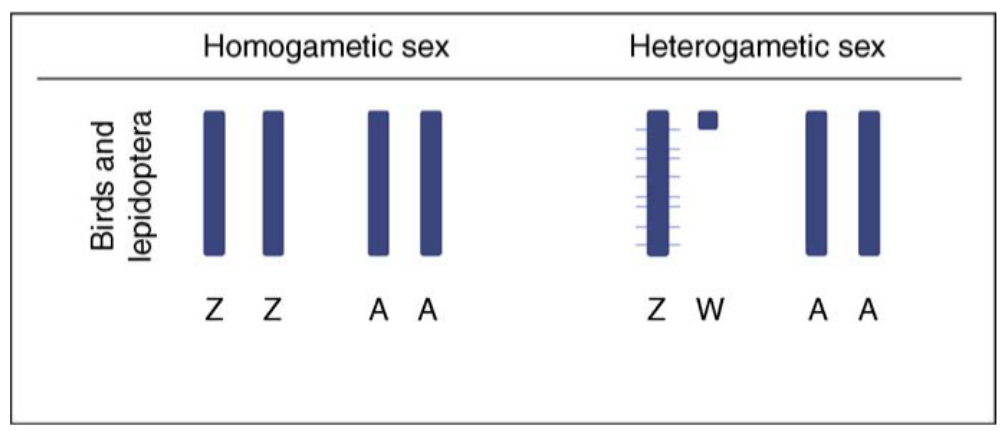

Figure 2.

Local dosage compensation in birds and Lepidopterans. In females (ZW), gene expression levels for some genes are hyper-transcribed locally, and the remaining genes are expressed normally. This produces an overall Z:A transcription ratio $\approx 0.8$ in females. Gene expression levels are indicated by chromosome width, with the expected normal expression level indicated in dark blue, and hyper-transcription above this level indicated in light blue. 


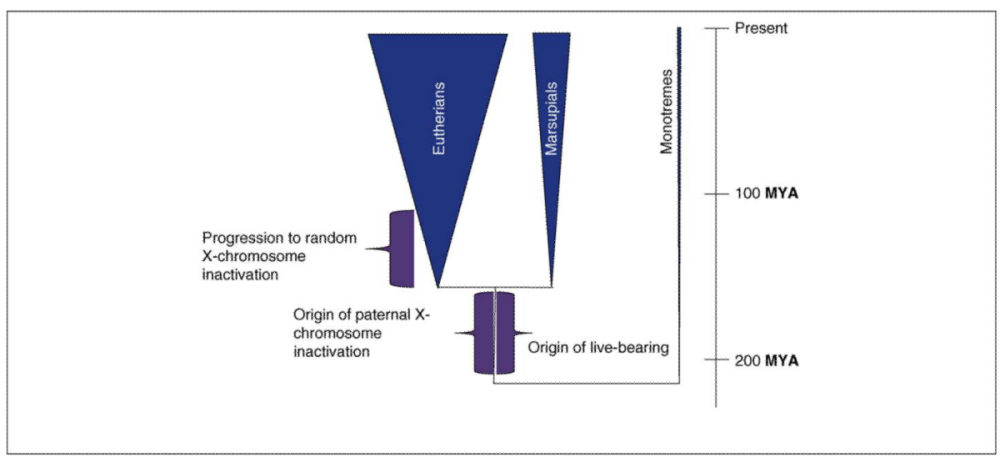

Figure 3.

The evolution of internal gestation and X-chromosome inactivation in mammals. Between 180 and 210 MYA, the evolution of internal gestation led to the origin of live-bearing in the therian mammals (marsupials and eutherians). X-chromosome inactivation also originated on the branch separating the monotremes and therians, and at first involved only the paternal copy. After the divergence of the eutherians, this inactivation progressed to include either copy of the X, randomly determined in each cell early in development. Triangle width corresponds approximately to species diversity within the three mammalian clades. 


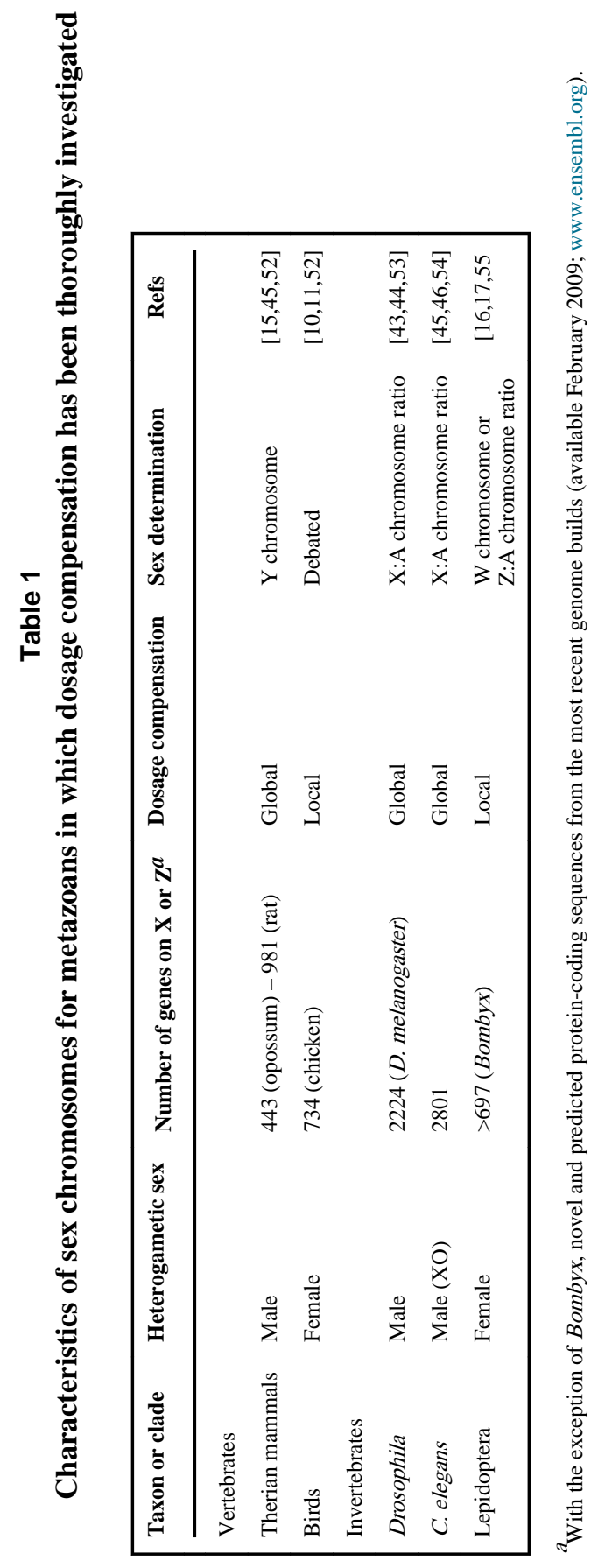

Trends Genet. Author manuscript; available in PMC 2010 August 17. 